\title{
The first case of vaginal angiomatoid Spitz nevus causing vaginal bleeding
}

\author{
Yong Hee Park ${ }^{1}$, Jung Mi Byun ${ }^{1,2}$, Hwa Jin Cho ${ }^{3}$, Dae Hoon Jeong ${ }^{1,2}$, Young Nam Kim ${ }^{1,2}$, Hye Rim Park ${ }^{1}$,
} Kyung Bok Lee ${ }^{1,2}$, Moon Su Sung ${ }^{1,2}$

${ }^{1}$ Department of Obstetrics and Gynecology, ${ }^{2}$ Paik Institute for Clinical Research, ${ }^{3}$ Department of Pathology, Inje University Busan Paik Hospital, Busan, Korea

Angiomatoid Spitz nevus is a variant of melanocytic nevus with prominent vasculature. Due to its pathologic features, angiomatoid Spitz nevus in the vaginal wall is extremely rare. A 42-year-old woman presented to the hospital with abnormal vaginal bleeding. Vaginal examination revealed a $2 \times 2-\mathrm{cm}$ well-demarcated tumor on the posterior wall of the vagina. The mass was successfully removed by complete excision and was diagnosed as angiomatoid Spitz nevus on pathologic examination. We present the first reported case of vaginal angiomatoid Spitz nevus, which caused vaginal bleeding. Although angiomatoid Spitz nevus has many histopathological similarities with malignant melanoma, precise histopathological diagnosis is important for preventing overtreatment.

Keywords: Vaginal bleeding; Angiomatoid Spitz nevus; Melanoma

\section{Introduction}

Spitz nevus is a type of melanocytic nevus composed of spindle and epithelioid cells. Since the first description of Spitz nevus in 1948 [1,2], variants of Spitz nevus, such as desmoplastic, hyalinizing, pagetoid, plexiform, myxoid, polypoid, tubular epithelioid cell, and angiomatoid Spitz nevus have been reported. Angiomatoid Spitz nevus was first described by Diaz-Cascajo et al. [1] as a subtype of desmoplastic Spitz nevus with prominent vasculature. It is a subtype of melanocytic nevus and usually presents as a solitary nodule on the skin of the extremities or the face in young adults or children [1-3]. It is very difficult to identify angiomatoid Spitz nevus on the mucosal areas, such as the vagina or oral cavity, because usually there are few melanocytes in the mucosal epithelium.

We report an extremely rare case of a 42-year-old woman with abnormal vaginal bleeding from a vaginal wall neoplastic tumor that was surgically resected and ultimately diagnosed as angiomatoid Spitz nevus.

\section{Case report}

A 42-year-old parous woman presented to our hospital with a 7-day history of persistent vaginal bleeding. Her menstrual cycle was very regular, and her last period was 2 weeks prior. She had multiple leiomyomas identified by transvaginal ultrasound 5 years prior and follow-up investigations had been performed. On pelvic examination, the uterus appeared slightly enlarged, and a $2 \times 2-\mathrm{cm}$ well-demarcated mass was detected on the posterior upper wall of the vagina. Rectal examination showed no abnormalities. Transvaginal ultrasound showed a $7 \times 10-\mathrm{cm}$ uterus with multiple leiomyomas and a $2 \times 2-\mathrm{cm}$ hypoechogenic mass on the superior vaginal wall. Total laparoscopic hysterectomy and vaginal wall mass excision were performed, and several intramural and subserosal

Received: 2018.07.15. Revised: 2018.08.12. Accepted: 2018.08.22. Corresponding author: Jung Mi Byun

Department of Obstetrics and Gynecology, Inje University Busan Paik Hospital, 75 Bokji-ro, Busanjin-gu, Busan 47392, Korea

E-mail: hellojungmi@hanmail.net

https://orcid.org/0000-0002-5570-3606

Articles published in Obstet Gynecol Sci are open-access, distributed under the terms of the Creative Commons Attribution Non-Commercial License (http://creativecommons. org/licenses/by-nc/3.0/) which permits unrestricted non-commercial use, distribution, and reproduction in any medium, provided the original work is properly cited.

Copyright $\odot 2019$ Korean Society of Obstetrics and Gynecology 


\title{
Obstetrics \& Gynecology Science
}

\author{
Yong Hee Park, et al. Vaginal angiomatoid Spitz nevus
}

leiomyomas were identified on the uterus. Under laparoscopy, we visualized a $2 \times 2 \times 1-\mathrm{cm}$ mass with abundant blood vessels on the posterior upper part of the vagina (Fig. 1). The mass was completely removed with clear surgical margins.

Microscopic examination of the vaginal mass revealed a dome-shaped angiomatoid Spitz nevus with a well-defined margin and several small vessels on the surface. Histologic examination revealed that the tumor consisted of hypercellular epithelioid melanocytes and variable sized vessels (Fig. 2A) and tumor nests composed of dermis tissue with collagen bands (Fig. 2B). Cohesive clusters of uniform round cells with scant cytoplasm surrounding vessels and prominent endothelial cells protruding into the vessels were observed (Fig.

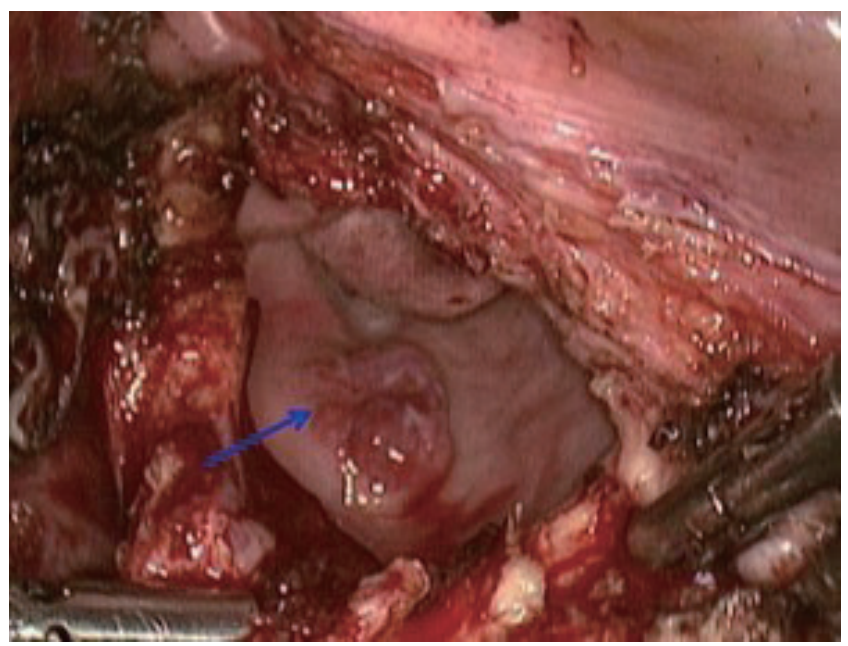

Fig. 1. Laparoscopic images of the lesion. After laparoscopic hysterectomy, the vaginal mass (blue arrow) visible through the vaginal orifice. Gross examination showing a $2 \times 2 \times 1-\mathrm{cm}$ mass with abundant blood vessels on the surface of the posterior upper part of the vagina.
2B). The melanocytes had minimal nuclear pleomorphism, indistinct nucleoli, and no prominent mitosis or necrosis. Immunohistochemical staining indicated strong positivity of the nevus cells for S-100 (Fig. 2C). Only 5\% of the cells stained positive for Ki-67, signifying low malignant potential. CD34 was strongly expressed in the blood vessels (Fig. 2D).

The patient had an uneventful operative course and was discharged on postoperative day 4. During follow-up, there was no evidence of local recurrence and distant metastasis up to 3 years after the operation.

\section{Discussion}

We report an extremely rare case of angiomatoid Spitz nevus in a 42-year old woman who experienced persistent vaginal bleeding for 7 days. The lesion was surgically removed and diagnosed as angiomatoid Spitz nevus, based on histopathological findings. Angiomatoid Spitz nevus usually presents as an intradermal lesion without involvement of the epidermis $[1,3]$. Neoplastic melanocytes show epithelioid or spindle features, as in typical Spitz nevus, and possess normochromatic nuclei with abundant eosinophilic cytoplasm. Thick collagen bundles are present on tumor nests $[1,3]$.

In our case, the lesion was formed by soft tissue with a proliferation of small blood vessels; thus, it could easily have been misdiagnosed as a hemangioma or a malignant tumor. Angiomatoid Spitz nevi show many small vessels in their stroma. Contrary to malignant lesions, vessels are abundant in the upper part of the lesion $[1,3,4]$. The vessels form clusters or are distributed as solitary units. Some lesions extended into the subcutaneous junction. There is a difference
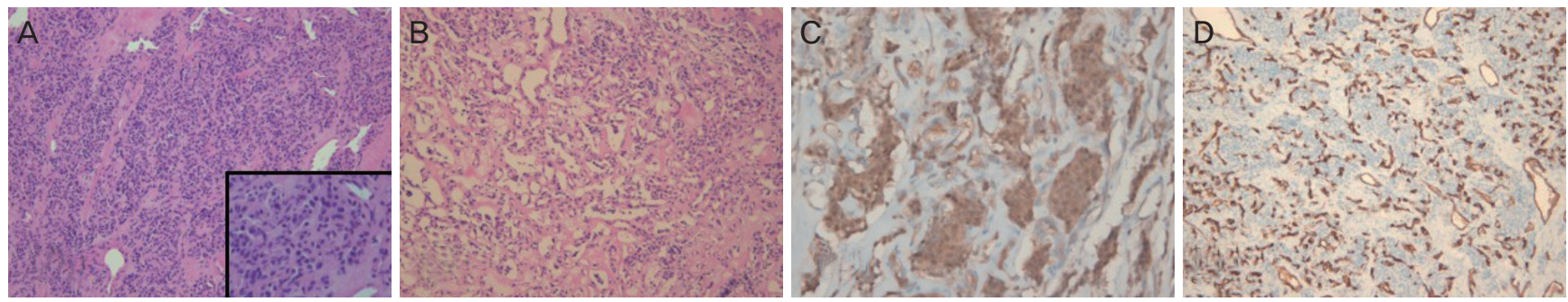

Fig. 2. Pathologic findings. (A) Dermis with hypercellular epithelioid melanocytes and variable sized vessels (hematoxylin and eosin [H\&E] stain, $\times 200)$. Inlet: cohesive clusters of uniform round tumor cells with scant cytoplasm, minimal nuclear pleomorphism, and indistinct nucleoli (H\&E stain, $\times 400)$. (B) Thin to thick bands of hyalinized collagen throughout the dermis. Prominent endothelial cells protruding into the vessels. (C) Diffuse positivity of the nevus cells for S100 (S100 stain). (D) CD34 expression is indicative of prominent vasculature (CD34 stain). 


\section{Obstetrics \& Gynecology Science}

Vol. 62, No. 4, 2019

between telangiectasias of other variants of Spitz nevus and angiomatoid Spitz nevus, because blood vessels of angiomatoid Spitz nevus are larger in number and smaller in diameter. In addition, they show thick walls and small round lumina lined by plump endothelial cells without cellular atypia [4].

Clinically, most angiomatoid Spitz nevi are reported as lesions of the extremities of young adults $[2,3,5]$. Vaginal angiomatoid Spitz nevus is extremely rare. Al-Shraim [6] reported a similar case of angiomatoid giant cellular blue nevus of the vaginal wall. Compared to that reported by Al-Shraim [6], the lesion in our case should strong immunohistochemical positivity for CD34. Our case of angiomatoid Spitz nevus with vaginal bleeding is the first such report in Korea. Melanocytes are usually present in the basal layer of the epidermis of the skin, but are also found in the basal portion of the epidermis of the vaginal mucosa in 3\% of women as an embryological remnant of the neural crest cells. Spitz nevi of the vagina develops from aberrantly located melanocytes within the vaginal mucosa epidermis [5].

The most important differential diagnosis of angiomatoid Spitz nevus is malignant melanoma. Malignant melanoma of the vagina may also originate from aberrant melanocytes and has similar histopathological features [5]. Due to the architectural similarity, Spitz nevus are often misdiagnosed as regressing malignant melanoma [7]. However, angiomatoid Spitz nevus does not extend beyond the epidermis and has no prominent cellular atypia or mitotic activity $[1,2]$. Thus, angiomatoid Spitz nevus shows very weak immunohistochemical staining for Ki-67, a proliferation index. Vaginal malignant melanoma often shows local recurrence or distant metastasis and is aggressive in nature; therefore, patients need additional surgical treatment, such as wide excision or lymph node dissection, chemotherapy, or radiotherapy [8]. In contrast, the clinical course of Spitz nevus is invariably benign. Thus, identification of the correct condition between malignant vaginal melanoma and angiomatoid Spitz nevus is important for treatment.

In conclusion, to the best of our knowledge, this is the first reported case of vaginal angiomatoid Spitz nevus with abnormal vaginal bleeding in Korea. Although angiomatoid Spitz nevus has many histopathological similarities with malignant melanoma, the prognosis is very different for the 2 diseases. Therefore, precise histopathological diagnosis is important for preventing overtreatment.

\section{Conflict of interest}

No potential conflict of interest relevant to this article was reported.

\section{Ethical approval}

The study was approved by the Institutional Review Board of Inje University Busan Paik Hospital (IRB No. 17-0101) and performed in accordance with the principles of the Declaration of Helsinki.

\section{Patient consent}

The patients provided written informed consent for the publication and the use of their images.

\section{References}

1. Diaz-Cascajo C, Borghi S, Weyers W. Angiomatoid Spitz nevus: a distinct variant of desmoplastic Spitz nevus with prominent vasculature. Am J Dermatopathol 2000;22:135-9.

2. Spitz S. Melanomas of childhood. Am J Pathol 1948;24:591-609.

3. Fabrizi G, Massi G. Angiomatoid Spitz naevus: a close simulator of regressing malignant melanoma. $\mathrm{Br} J$ Dermatol 2001;145:845-6.

4. Tetzlaff MT, Xu X, Elder DE, Elenitsas R. Angiomatoid Spitz nevus: a clinicopathological study of six cases and a review of the literature. J Cutan Pathol 2009;36:4716.

5. Gökaslan H, Sişmanoğlu A, Pekin T, Kaya H, Ceyhan N. Primary malignant melanoma of the vagina: a case report and review of the current treatment options. Eur J Obstet Gynecol Reprod Biol 2005;121:243-8.

6. Al-Shraim MM. Angiomatoid giant cellular blue nevus of vaginal wall associated with pregnancy. Diagn Pathol 2011;6:32.

7. Magro CM, Crowson AN, Mihm MC. Unusual variants of malignant melanoma. Mod Pathol 2006;19 Suppl 2:S41-70. 


\section{Obstetrics \& Gynecology Science}

Yong Hee Park, et al. Vaginal angiomatoid Spitz nevus

8. Androutsopoulos G, Terzakis E, loannidou G, Tsamandas A, Decavalas G. Vaginal primary malignant melanoma: a rare and aggressive tumor. Case Rep Obstet Gynecol 2013;2013:137908. 